\title{
New soliton solutions of dissipative (2+1)-dimensional AKNS equation
}

\author{
Somayeh Arbabi , Mohammad Najafi* , Malihe Najafi \\ Department of Mathematics, Anar Branch, Islamic Azad University, Anar, Iran \\ *Corresponding author E-mail: m_najafi82@yahoo.com
}

\begin{abstract}
We employ the idea of Hirota's bilinear method, to obtain some new exact soliton solutions for high nonlinear form of dissipative (2+1)-dimensional AKNS equation. Multiple singular soliton solutions were obtained by this method. Moreover, multiple singular soliton solutions were also derived.
\end{abstract}

Keywords: Hirota bilinear method, dissipative (2+1)-dimensional AKNS equation, Multiple soliton solutions, Multiple singular soliton solutions.

\section{Introduction}

Nonlinearity is a fascinating element of nature, today, many scientists see nonlinear science as the most important frontier for the fundamental understanding of nature. Many complex physical phenomena are frequently described and modeled by nonlinear evolution equation, so the exact or analytical solutions of the discussed nonlinear evolution equation become more and more important, which is considered not only a valuable tool in checking the accuracy of computational dynamics, but also gives us a good help to readily understand the essentials of complex physical phenomenon, e.g., collision of two solitary solutions. Looking for exact solitary wave solutions to nonlinear evolution equations has long been a major concern for both mathematicians and physicists. These solutions may well describe various phenomena in physics and other fields, such as solitons and propagation with a finite speed, and thus they may give more insight into the physical aspects of the problems. Modern theories of nonlinear science have been highly developed over the last half century.

The study of exact solutions of nonlinear evolution equations plays an important role in soliton theory and explicit formulas of such exact solutions play an essential role in the nonlinear science. Also, the explicit formulas may provide physical information and help us to understand the mechanism of related physical models. In recent years, many kinds of powerful methods have been proposed to find solutions of nonlinear partial differential equations, numerically and/or analytically, e.g., Binary Bell Polynomials Method [1], the multiple exp-function method [2], the tanh method [3], the sine-cosine method [4], the Exp-function method [5], the homogeneous balance method [6] and the extended homoclinic test approach [7].

In this paper, by means of the Hirota's bilinear method, we will obtain some exact and new solutions for the dissipative $(2+1)$-dimensional AKNS equation. In the following section we have a brief review on the Hirota's bilinear method and in Section 3 and 4, we apply the Hirota's bilinear method to obtain multiple soliton solutions and multiple singular soliton solutions of the dissipative (2+1)-dimensional AKNS equation. Finally, the paper is concluded in Section 5.

\section{The Hirota bilinear method}

To formally derive N-soliton solutions for completely integrable equations, we will use the Hirota's direct method combined with the simplified version of [8-10]. It was proved by many that soliton solutions are just polynomials of exponentials. This will be also confirmed in the coming discussions. We first substitute $u(x, y, t)=e^{k x+m y-w t}$

into the linear terms of any equation under discussion to determine the relation between $k, m$ and $w$. We then substitute the Cole-Hopf transformation $u(x, y, t)=R(\ln f(x, y, t))_{x}$, 
into the equation under discussion, where the auxiliary function $f$, for the single soliton solution, is given by $f(x, y, t)=1+C_{1} f_{1}(x, y, t)=1+C_{1} e^{\theta_{1}}$.

The steps of the Hirota's method as summarized in [11-14] are as follows:

(i) For the relation between $k_{i}, m_{i}$ and $w_{i}$, we use

$u(x, y, t)=e^{\theta_{i}}, \theta_{i}=k_{i} x+m_{i} y-w_{i} t$,

(ii) For single soliton, we use

$f=1+C_{1} e^{\theta_{1}}$,

to determine $\mathrm{R}$.

(iii) For two-soliton solutions, we use

$f=1+C_{1} e^{\theta_{1}}+C_{2} e^{\theta_{2}}+C_{1} C_{2} a_{12} e^{\theta_{1}+\theta_{2}}$,

to determine the phase shift coefficient $a_{12}$, and hence can be generalized for $a_{i j}, 1 \leq i<j \leq 3$.

(iv) For three-soliton solutions, we use

$f=1+C_{1} e^{\theta_{1}}+C_{2} e^{\theta_{2}}+C_{3} e^{\theta_{3}}+C_{1} C_{2} a_{12} e^{\theta_{1}+\theta_{2}}+C_{1} C_{3} a_{13} e^{\theta_{1}+\theta_{3}}+C_{2} C_{3} a_{23} e^{\theta_{2}+\theta_{3}}+C_{1} C_{2} C_{3} b_{123} e^{\theta_{1}+\theta_{2}+\theta_{3}}$,

to determine $b_{123}$. Pekcan proved in [15], $b_{123}=a_{12} a_{23} a_{13}$, then the equation gives rise to three-soliton solutions.

In the following, we will apply the aforementioned steps to Boiti-Leon-Manna-Pempinelli equation. Multiple soliton solutions are obtained for $C_{1}=C_{2}=C_{3}=1$. However, multiple singular soliton solutions are obtained if $C_{1}=C_{2}=C_{3}=-1$.

\section{Multiple soliton solutions of dissipative (2+1)-dimensional AKNS equation}

In this paper, we investigate explicit formula of soliton solutions of the following high nonlinear form of dissipative (2+1)-dimensional AKNS equation [1]:

$4 u_{x t}+8 u_{x y} u_{x}+4 u_{x x} u_{y}+u_{x x y}+\beta u_{x x}=0$.

To determine multiple-soliton solutions for Eq. (8), we follow the steps presented above. We first consider $C_{1}=C_{2}=C_{3}=1$. Substituting

$u(x, y, t)=e^{\theta_{i}}, \theta_{i}=k_{i} x+m_{i} y-w_{i} t$,

into the linear terms of Eq.(8) to find the relation

$w_{i}=\frac{1}{4} k_{1}^{2} m_{1}+\frac{1}{4} \beta k_{1}, \quad i=1,2, \ldots, N$

and consequently, $\theta_{i}$ becomes

$\theta_{i}=k_{i} x+m_{i} y-\left(\frac{1}{4} k_{1}^{2} m_{1}+\frac{1}{4} \beta k_{1}\right) t$.

To determine $R$, we substitute

$u(x, y, t)=R(\ln f(x, y, t))_{x}$,

where

$f(x, y, t)=1+f_{1}(x, y, t)=1+e^{k_{1} x+m_{1} y-\left(\frac{1}{4} k_{1}^{2} m_{1}+\frac{1}{4} \beta k_{1}\right) t}$

into Eq.(8) and solve to find that $R=1$.

This means that the single singular soliton solution is given by

$u(x, y, t)=\frac{k_{1} e^{k_{1} x+m_{1} y-\left(\frac{1}{4} k_{1}^{2} m_{1}+\frac{1}{4} \beta k_{1}\right) t}}{1+e^{k_{1} x+m_{1} y-\left(\frac{1}{4} k_{1}^{2} m_{1}+\frac{1}{4} \beta k_{1}\right) t}}$.

For the two-soliton solutions, we substitute

$u(x, y, t)=(\ln f(x, y, t))_{x}$,

where

$f(x, y, t)=1+e^{\theta_{1}}+e^{\theta_{2}}+a_{12} e^{\theta_{1}+\theta_{2}}$,

into Eq.(8), where $\theta_{1}$ and $\theta_{2}$ are given in Eq.(11) to obtain 
$a_{12}=\frac{\left(k_{1}-k_{2}\right)^{2}}{\left(k_{1}+k_{2}\right)^{2}}$

and $w_{s}=\frac{1}{4} k_{s}^{2} m_{s}+\frac{1}{4} \beta k_{s}, s=1,2$.

for $\left|k_{1}\right| \neq\left|k_{2}\right|$, hence

$a_{i j}=\frac{\left(k_{i}-k_{j}\right)^{2}}{\left(k_{i}+k_{j}\right)^{2}}$,

for $\left|k_{i}\right| \neq\left|k_{j}\right|$. This in turn gives

$f(x, y, t)=1+e^{\theta_{1}}+e^{\theta_{2}}+\frac{\left(k_{1}-k_{2}\right)^{2}}{\left(k_{1}+k_{2}\right)^{2}} e^{\theta_{1}+\theta_{2}}$,

where

$\theta_{i}=k_{i} x+m_{i} y-\left(\frac{1}{4} k_{i}^{2} m_{i}+\frac{1}{4} \beta k_{i}\right) t, i=1,2$,

which is a two soliton solution(Fig. 1).

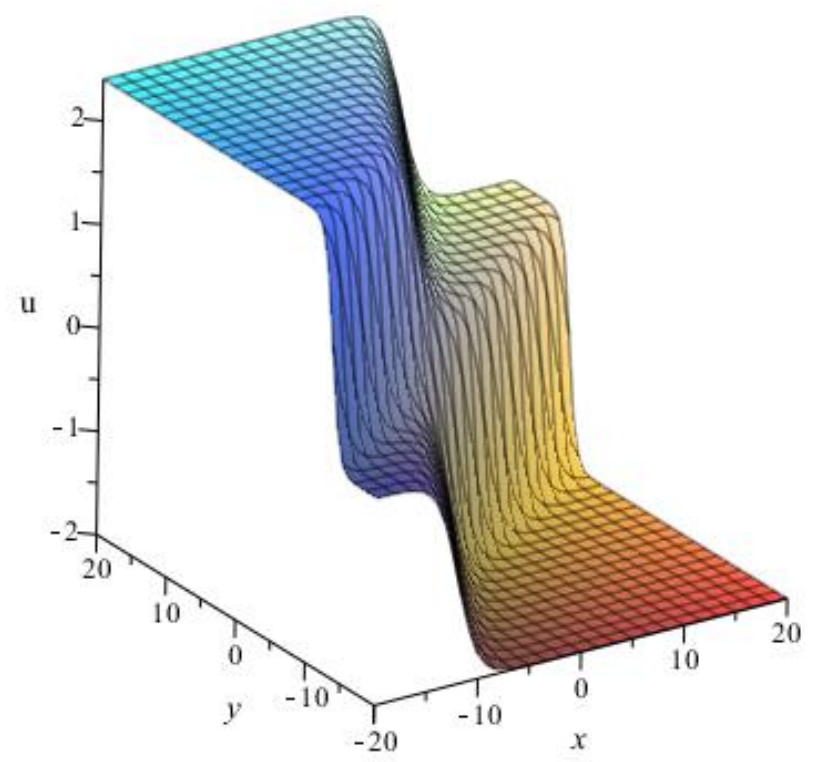

Fig.1: The two soliton solution with $k_{1}=1, m_{1}=0.6, k_{2}=-1.2$ and $m_{2}=1.8$.

Similarly, to determine the three soliton solutions, we set

$f(x, y, t)=1+e^{\theta_{1}}+e^{\theta_{2}}+e^{\theta_{3}}+a_{12} e^{\theta_{1}+\theta_{2}}+a_{13} e^{\theta_{1}+\theta_{3}}+a_{23} e^{\theta_{2}+\theta_{3}}+a_{12} a_{23} a_{13} e^{\theta_{1}+\theta_{2}+\theta_{3}}$.

To determine the three soliton solutions explicitly, we substitute the last result for $f(x, y, t)$ into Eqs. (28), (See Fig. 2).

The higher level soliton solutions, for $n \geq 4$ can be obtained in a parallel manner. The obtained results confirm that the dissipative (2+1)-dimensional AKNS equation is completely integrable and possesses multiple soliton solutions of any order. 


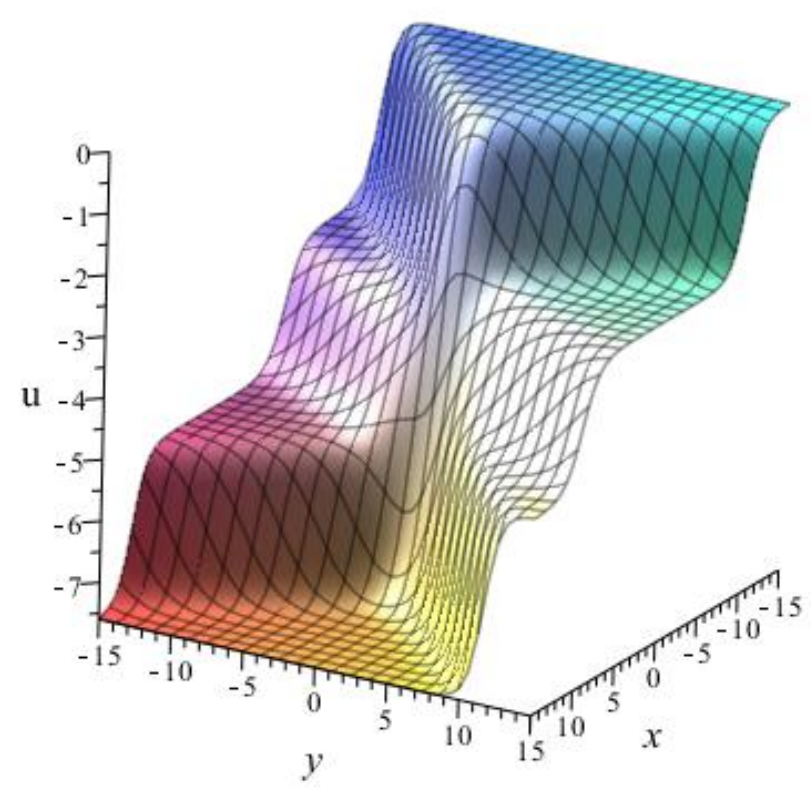

Fig. 2: The three soliton solution with $k_{1}=1, m_{1}=-0.6, k_{2}=1.2, m_{2}=0.8, k_{3}=-1.8$ and $m_{3}=-1.8$.

\section{Multiple singular soliton solutions of dissipative (2+1)-dimensional AKNS equation}

We first consider $C_{1}=C_{2}=C_{3}=-1$. Substituting

$u(x, y, t)=e^{\theta_{i}}, \theta_{i}=k_{i} x+m_{i} y-w_{i} t$,

into the linear terms of Eq.(8) to find the relation

$w_{i}=\frac{1}{4} k_{1}^{2} m_{1}+\frac{1}{4} \beta k_{1}, \quad i=1,2, \ldots, N$

and consequently, $\theta_{i}$ becomes

$\theta_{i}=k_{i} x+m_{i} y-\left(\frac{1}{4} k_{1}^{2} m_{1}+\frac{1}{4} \beta k_{1}\right) t$.

To determine $R$, we substitute

$u(x, y, t)=R(\ln f(x, y, t))_{x}$,

where $f(x, y, t)=1-f_{1}(x, y, t)=1-e^{k_{1} x+m_{1} y-\left(\frac{1}{4} k_{1}^{2} m_{1}+\frac{1}{4} \beta k_{1}\right) t}$ into Eq.(8) and solve to find that $R=1$. .

This means that the single singular soliton solution is given by

$u(x, y, t)=\frac{k_{1} e^{k_{1} x+m_{1} y-\left(\frac{1}{4} k_{1}^{2} m_{1}+\frac{1}{4} \beta k_{1}\right) t}}{1-e^{k_{1} x+m_{1} y-\left(\frac{1}{4} k_{1}^{2} m_{1}+\frac{1}{4} \beta k_{1}\right) t}}$.

For the two-soliton solutions, we substitute

$u(x, y, t)=(\ln f(x, y, t))_{x}$,

where

$f(x, y, t)=1-e^{\theta_{1}}-e^{\theta_{2}}+a_{12} e^{\theta_{1}+\theta_{2}}$,

into Eq.(8), where $\theta_{1}$ and $\theta_{2}$ are given in Eq.(25) to obtain

$a_{12}=\frac{\left(k_{1}-k_{2}\right)^{2}}{\left(k_{1}+k_{2}\right)^{2}}$, 
and $w_{s}=\frac{1}{4} k_{s}^{2} m_{s}+\frac{1}{4} \beta k_{s}, s=1,2$.

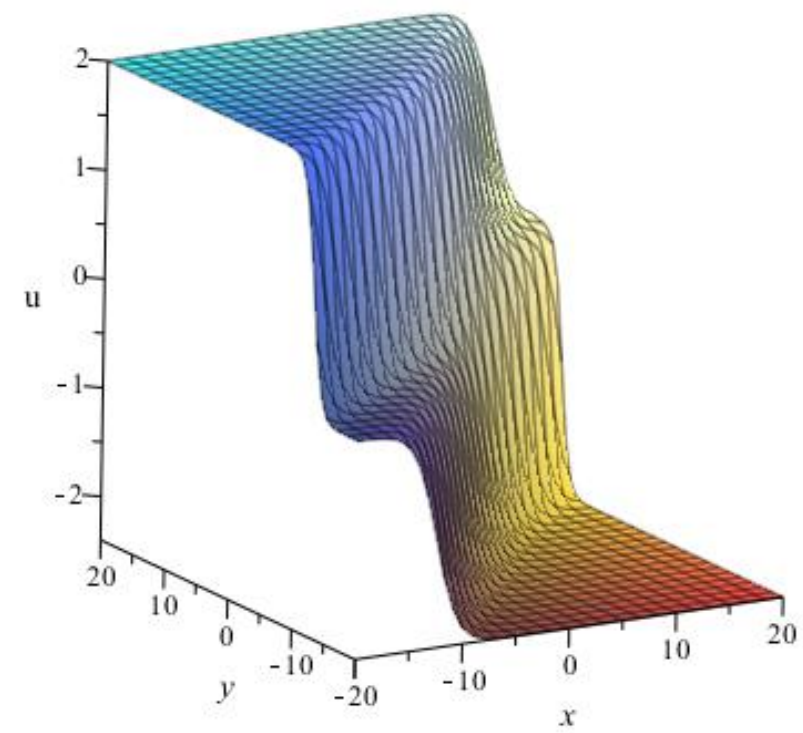

Fig. 3: The two soliton solution with $k_{1}=-1, m_{1}=0.8, k_{2}=1.2$ and $m_{2}=-1.6$.

For $\left|k_{1}\right| \neq\left|k_{2}\right|$, hence

$a_{i j}=\frac{\left(k_{i}-k_{j}\right)^{2}}{\left(k_{i}+k_{j}\right)^{2}}$,

for $\left|k_{i}\right| \neq\left|k_{j}\right|$. This in turn gives

$f(x, y, t)=1-e^{\theta_{1}}-e^{\theta_{2}}+\frac{\left(k_{1}-k_{2}\right)^{2}}{\left(k_{1}+k_{2}\right)^{2}} e^{\theta_{1}+\theta_{2}}$,

where

$\theta_{i}=k_{i} x+m_{i} y-\left(\frac{1}{4} k_{i}^{2} m_{i}+\frac{1}{4} \beta k_{i}\right) t, i=1,2$,

which is a two soliton solution(Fig. 3).

Similarly, to determine the three soliton solutions, we set

$f(x, y, t)=1-e^{\theta_{1}}-e^{\theta_{2}}-e^{\theta_{3}}+a_{12} e^{\theta_{1}+\theta_{2}}+a_{13} e^{\theta_{1}+\theta_{3}}+a_{23} e^{\theta_{2}+\theta_{3}}-a_{12} a_{23} a_{13} e^{\theta_{1}+\theta_{2}+\theta_{3}}$.

To determine the three soliton solutions explicitly, we substitute the last result for $f(x, y, t)$ into Eqs. (28), ( See Fig. 4).

The higher level singular soliton solutions, for $n \geq 4$ can be obtained in a parallel manner.

\section{Conclusion}

In this paper, by using the Hirota bilinear method, we obtained some explicit formulas of solutions for the dissipative (2+1)-dimensional AKNS equation. Multiple soliton solutions were formally derived. Moreover, multiple singular soliton solutions of any order was derived as well. The results of other works are special cases of our results. 


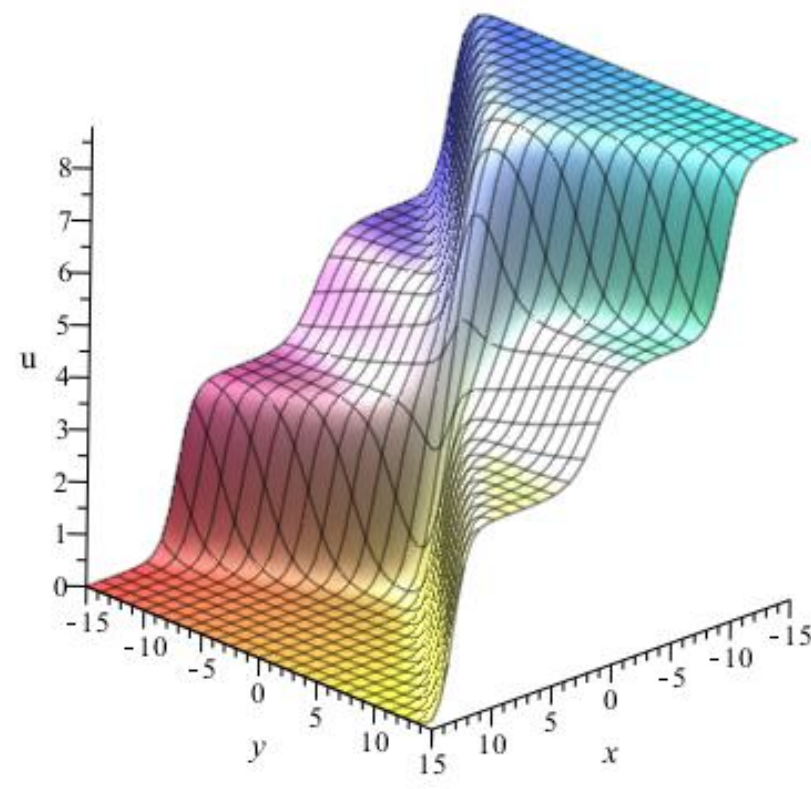

Fig. 4: The three soliton solution with $k_{1}=-1, m_{1}=0.2, k_{2}=-1.6, m_{2}=0.8, k_{3}=-1.8$ and $m_{3}=1.6$.

\section{References}

[1] N. LIU, X. Q. LIU, Application of the Binary Bell Polynomials Method to the Dissipative (2+1)-Dimensional AKNS Equation, Chin. Phys. Lett., 29 (2012) 120-201

[2] M. T. Darvishi, M. Najafi, L. Kavitha L, M. Venkatesh, Stair and Step Soliton Solutions of the Integrable (2+1) and (3+1)-Dimensional BoitiLeon-Manna-Pempinelli Equations, Commun. Theor. Phys. 58 (2012) 785-794.

[3] A.M. Wazwaz, The extended tanh method for new solitons solutions for many forms of the fifth- order KdV equations, Appl. Math. Comput. 184 (2007) 1002-1014.

[4] A.M. Wazwaz, A sine-cosine method for handling nonlinear wave equations, Math. Comput. Model. 40 (2004) $499-508$.

[5] A. Borhanifar, M.M Kabir , New periodic and soliton solutions by application of Exp-function method for nonlinear evolution equations. Journal of Computational and Applied Mathematics, 229 (2009) 158-167.

[6] Z. Xiqiang, W. Limin, S. Weijun, The repeated homogeneous balance method and its applications to nonlinear partial differential equations, Chaos, Solitons and Fractals, 28 (2006) 448-453.

[7] M.T. Darvishi, M. Najafi, A modification of extended homoclinic test approach to solve the (3+1)-dimensional potential-YTSF equation, Chin. Phys. Lett., 28 (2011) 040202.

[8] W. Hereman, W Zhuang, Symbolic software for soliton theory. Acta Applicandae Mathematicae Phys Lett A 76 (1980) 95-96.

[9] W. Hereman, W Zhuang, A MACSYMA program for the Hirota method. 13th World Congress Comput Appl Math , 2 (1991) 842-863.

[10] W. Hereman, A Nuseir, Symbolic methods to construct exact solutions of nonlinear partial differential equations. Math Comput Sim , 43 (1997) 13-27.

[11] A.M. Wazwaz, Regular soliton solutions and singular soliton solutions for the modified Kadomtsev-Petviashvili equations. Appl Math Comput, 204 ( 2008) 817-823.

[12] A.M. Wazwaz, A $(3+1)$-dimensional nonlinear evolution equation with multiple soliton solutions and multiple singular soliton solutions. Appl Math Comput, 215 (2009) 1548-1552.

[13] A.M. Wazwaz, New solitons and kink solutions for the Gardner equation. Commun Nonlinear Sci Numer Simul, 12 (2007) $1395-1404$.

[14] A.M. Wazwaz, Multiple soliton solutions for the (2+1)-dimensional asymmetric Nizhanik-Novikov-Veselov equation. Nonlinear Anal Ser A: Theory Meth Appl, 72 (2010) 1314-1318.

[15] Pekcan A. The Hirota Direct Method (a thesis master of science). Bilkent university 2005. 